(c) 2006 International Press

Adv. Theor. Math. Phys. 10 (2006) 683-712

\title{
Geometric quantization and non-perturbative Poisson sigma model
}

Francesco Bonechi ${ }^{1}$, Alberto S. Cattaneo ${ }^{2}$, and Maxim Zabzine ${ }^{3}$

${ }^{1}$ I.N.F.N. and Dipartimento di Fisica Via G. Sansone 1, 50019 Sesto Fiorentino - Firenze, Italy

francesco.bonechi@fi.infn.it

${ }^{2}$ Institut für Mathematik, Universität Zürich-Irchel Winterthurerstrasse 190, CH-8057 Zürich, Switzerland alberto.cattaneo@math.unizh.ch

${ }^{3}$ School of Mathematical Sciences, Queen Mary, University of London Mile End Road, London E1 4NS, UK m.zabzine@qmul.ac.uk

\begin{abstract}
In this note, we point out the striking relation between the conditions arising within geometric quantization and the non-perturbative Poisson sigma model. Starting from the Poisson sigma model, we analyze necessary requirements on the path integral measure which imply a
\end{abstract}

e-print archive: http://arXiv.org/abs/math.sg/0507223 
certain integrality condition for the Poisson cohomology class $[\alpha]$. The same condition was considered before by Crainic and Zhu but in a different context. In the case when $[\alpha]$ is in the image of the sharp map, we reproduce the Vaisman's condition for prequantizable Poisson manifolds. For integrable Poisson manifolds, we show, with a different procedure than in Crainic and Zhu, that our integrality condition implies the prequantizability of the symplectic groupoid. Using the relation between prequantization and symplectic reduction, we construct the explicit prequantum line bundle for a symplectic groupoid. This picture supports the program of quantization of Poisson manifold via symplectic groupoid. At the end, we discuss the case of a generic coisotropic $D$-brane.

\section{Introduction}

Quantization is generally understood as a transition from classical to quantum mechanics. In mathematics, a quantization of Poisson manifold should be a prescription able to produce a structure which physicists would agree to call the quantum theory associated with the classical system given by the Poisson manifold. However, this prescription is far from being unique and different people give a different meanings to the word quantization. In this note, we are interested in two different incarnations of the word quantization: geometric quantization and deformation quantization.

Deformation quantization deals with formal associative deformations of $C^{\infty}(M)$. In [16], Kontsevich gave a general formula for the deformation quantization of the algebra of functions on a Poisson manifold $(M, \alpha)$. Later in [7], it was established that the perturbative path integral expansion of the Poisson sigma model over the two-dimensional disk $D$ leads to the Kontsevich's star product.

The Poisson sigma model, introduced in $[14,20]$, is a topological twodimensional field theory with target a Poisson manifold $M$, whose Poisson tensor we will denote by $\alpha$ throughout. Let $\Sigma$ be a two-dimensional oriented compact manifold with a boundary. The starting point is the classical action functional $S$ defined on the space of vector bundle morphisms $\hat{X}: T \Sigma \rightarrow T^{*} M$ from the tangent bundle $T \Sigma$ to the cotangent bundle $T^{*} M$ of the Poisson manifold $M$. Such a map $\hat{X}$ is given by its base map $X: \Sigma \rightarrow M$ and the linear map $\eta$ between fibers, which may also be regarded as a section in $\Gamma\left(\Sigma, \operatorname{Hom}\left(T \Sigma, X^{*}\left(T^{*} M\right)\right)\right)$. The pairing $\langle$,$\rangle between the$ cotangent and tangent space at each point of $M$ induces a pairing between the differential forms on $\Sigma$ with values in the pull-backs $X^{*}\left(T^{*} M\right)$ and $X^{*}(T M)$, respectively. It is defined as pairing of the values and the exterior 
product of differential forms. Then, the action functional $S$ of the theory is

$$
S(X, \eta)=\int_{\Sigma}\langle\eta, d X\rangle+\frac{1}{2}\langle\eta,(\alpha \circ X) \eta\rangle .
$$

Here $\eta$ and $d X$ are viewed as one-forms on $\Sigma$, with the values in the pullback of the cotangent and tangent bundles of $M$ correspondingly. Thus, in local coordinates, we can rewrite the action (1.1) as follows:

$$
S(X, \eta)=\int_{D} \eta_{\mu} \wedge d X^{\mu}+\frac{1}{2} \alpha^{\mu \nu}(X) \eta_{\mu} \wedge \eta_{\nu}
$$

The variation of the action gives rise to the following equations of motion

$$
d \eta_{\rho}+\frac{1}{2}\left(\partial_{\rho} \alpha^{\mu \nu}\right) \eta_{\mu} \wedge \eta_{\nu}=0, \quad d X^{\mu}+\alpha^{\mu \nu} \eta_{\nu}=0
$$

In covariant language, these equations are equivalent to the statement that the bundle morphism $\hat{X}$ is a Lie algebroid morphism from $T \Sigma$ (with standard Lie algebroid structure) to $T^{*} M$ (with Lie algebroid structure canonically induced by the Poisson structure). The action (1.2) is invariant under the infinitesimal gauge transformations

$$
\delta_{\beta} X^{\mu}=\alpha^{\mu \nu} \beta_{\nu}, \quad \delta_{\beta} \eta_{\mu}=-d \beta_{\mu}-\left(\partial_{\mu} \alpha^{\nu \rho}\right) \eta_{\nu} \beta_{\rho},
$$

which form a closed algebra only on-shell (i.e., modulo the equations of motion (1.3)). We are interested in the situation when $\partial \Sigma \neq \emptyset$. Following [7], we first consider the boundary conditions

$$
\left.\eta_{t}\right|_{\partial \Sigma}=\left.0 \quad \beta\right|_{\partial \Sigma}=0,
$$

where $t$ corresponds to the direction tangent to the boundary. More general boundary conditions will be discussed in Section 5 .

In [7] it has been shown that the star product is given by the semiclassical expansion of the path integral of the Poisson sigma model over a disk $D$

$$
f * g(x)=\int_{X(\infty)=x} f(X(1)) g(X(0)) \mathrm{e}^{-(i / \hbar) S(X, \eta)} d X d \eta
$$

where $0,1, \infty$ are any three cyclically ordered points on the unit circle $\partial D$. The semiclassical expansion is to be understood as an expansion around the trivial classical solution $X=x$ and $\eta=0$. As it stands, the integral (1.6) is not well defined due to the gauge symmetries and renormalization. However, at perturbative level, this can be fixed [7].

In this note, we explore the idea that the formula (1.6) could make sense also outside of the perturbative expansion around the trivial solution. Although we do not know how to define the path integral non-perturbatively, in general, we can try to do different consistency checks. Thus, proposing different consistency tests for the Poisson sigma model on the sphere or on 
the disk with boundary conditions (1.5), we arrive to the following integrality condition, which is the main result of the paper:

$$
\frac{1}{2 \pi \hbar} S(X, \eta) \in \mathbb{Z}
$$

for every classical solution $X, \eta$ on the sphere or on the disk. We also show that this condition is equivalent to having, for every two-cycle $c_{2}$ which is the image of the base map of a Lie algebroid morphism $T \mathbb{S}^{2} \rightarrow T^{*} M$,

$$
\frac{1}{2 \pi \hbar} \int_{c_{2}} \omega_{\mathcal{L}} \in \mathbb{Z}
$$

where $\omega_{\mathcal{L}}$ denotes the induced symplectic form on the symplectic leaf $\mathcal{L}$ that contains $c_{2}$. This integrality condition is related to the different integrality conditions which appear within the geometric quantization program. It is also the necessary and sufficient condition for the symplectic groupoid of the given Poisson manifold to be prequantizable [11], a result that we will rederive, using different methods, in Section 4. The principal aim of this paper is to give different derivations of condition (1.8) and to relate it to the known integrality conditions. It appears that our considerations give strong support to the program [28] of quantizing Poisson manifolds via their corresponding symplectic groupoids. It seems plausible that considering the Poisson sigma model on any two-manifold $\Sigma$ should yield a stronger integrality condition; viz., (1.8) should be satisfied for any $c_{2}$ which is the image of the base map of a Lie algebroid morphism $T \Sigma \rightarrow T^{*} M$ for any $\Sigma$. However, we still do not have a proof of this fact. ${ }^{1}$

Moreover this integrality condition has a cohomological meaning. On a classical solution $\hat{X}=(X, \eta): T \Sigma \rightarrow T^{*} M(\partial \Sigma=\emptyset)$ the action functional can be written as

$$
S_{\mathrm{cl}}(X, \eta)=-\frac{1}{2} \int_{\Sigma}\langle\eta,(\alpha \circ X) \eta\rangle
$$

This expression depends only on the cohomology class of $[\alpha] \in H_{L P}^{\bullet}(M, \alpha)$ and the homology class of $(\Sigma, \hat{X})$ as explained in details in the Appendix; in fact (1.9) can be reintepreted in terms of a natural paring $\ll$, 》 between the Poisson cohomology $H_{L P}^{\bullet}(M, \alpha)$ and the homology whose cycles are algebroid morphisms. In particular it makes sense to talk of integral Poisson

\footnotetext{
${ }^{1}$ The first attempt to discuss the role of integrality in the context of PSM has been done in [21].
} 
tensor, which corresponds to requiring that

$$
\ll \alpha,\left(c_{2}, \hat{X}\right) \gg=2 \pi n \hbar, \quad n \in \mathbb{Z},
$$

for any algebroid morphism $\hat{X}: T c_{2} \rightarrow T^{*} M$, and, more generally, of integral Poisson cohomology, so that

$$
\left[\frac{1}{2 \pi \hbar} \alpha\right] \in H_{L P}^{2}(M, \alpha, \mathbb{Z}) .
$$

The organization of our presentation is as follows: In Section 2, we review the relevant concepts from the geometric quantization. In particular, we discuss the known integrability conditions on symplectic forms and Poisson tensors arising in geometric quantization. In Section 3, we consider the on-shell gauge transformations and the value of the action functional on classical solutions of the Poisson sigma model. We derive a generalized integrality condition for a Poisson tensor in subsection 3.1. In subsection 3.2, we interpret this condition in the symplectic case and discuss some peculiarities of the Poisson sigma model over symplectic manifolds. In subsection 3.3, we return to the case of general Poisson manifolds, discuss the cohomological nature of our integrality condition and recover Vaisman's condition for the prequantizability of the Poisson manifold as a special case. In Section 4, the integrality condition is rederived in a different context: Namely, we study the relation between symplectic reduction and prequantization in the context of the Poisson sigma model and its reduced phase space. Thus, assuming the integrality condition for an integrable Poisson manifold, we show that the corresponding symplectic groupoid is prequantizable. In Section 5, general boundary conditions are considered and the corresponding integrality conditions are found. In Section 6, we present concluding remarks with a possible interpretation of our results. In the Appendix, we collect the technicalities regarding the relevant (co)homology groups.

\section{Geometric quantization}

This section is just a reminder of some relevant elements of geometric quantization. Namely, we are going to review the necessary and sufficient conditions for the existence of prequantization bundles for symplectic and Poisson manifolds.

The geometric quantization theory of Kostant [17] and Souriau [24] was first developed for symplectic manifolds and then further generalized to Poisson manifolds. Within this approach, the quantization is done in two steps: prequantization which gives a linear representation of $\left(C^{\infty}(M),\{\},\right)$ 
by operators on a complex vector space and then quantization where one restricts to a convenient subalgebra of $\left(C^{\infty}(M),\{\},\right)$ (for review, see [15, $27,30])$.

We are interested in the first step of this construction. Modulo a certain obstruction, the prequantization problem can be solved by considering the space of sections of a complex line bundle $L \rightarrow M$, the prequantization bundle and a well-chosen prequantization formula. In other words, to each $f \in C^{\infty}(M)$ there corresponds an operator $\hat{f}$ acting on $\Gamma(L)$ such that the map $f \mapsto \hat{f}$ is linear and

$$
-i \hbar \widehat{\{f, g\}}=[\hat{f}, \hat{g}] \equiv \hat{f} \hat{g}-\hat{g} \hat{f}
$$

with $1 \rightarrow \hat{1}$.

Let us start from the symplectic case. Assuming that the line bundle $L$ exists, we can define $\hat{f}$ by

$$
\hat{f} s=-i \hbar \nabla_{X_{f}} s+f s,
$$

where $\nabla$ is a covariant derivative on $L$ and $X_{f}$ is the Hamiltonian vector field of $f$ (i.e., $X_{f} g=\{f, g\}$ for every function $g$ ). Then, (2.1) is equivalent to

$$
c_{1}(L)=-\frac{1}{2 \pi \hbar}[\omega]
$$

where $c_{1}(L)$ is the first Chern class of the line bundle $L$. Since the first Chern class is integral (i.e., $c_{1}(L) \in H^{2}(M, \mathbb{Z})$ ), one arrives at the following quantization condition for the symplectic form:

$$
\left[\frac{1}{2 \pi \hbar} \omega\right] \in H^{2}(M, \mathbb{Z})
$$

The converse is also true. Namely, if the symplectic form satisfies (2.4), then there exists the line bundle $L$ with connection $\nabla$ such that (2.1) and (2.2) are fulfilled. Observe that the representation $f \mapsto \hat{f}$ is faithful.

In the Poisson case, one can generalize the above construction [26]. The idea is that it is enough to define $\nabla$ only along the symplectic leaves and thus one needs only the partial connection on $L$. The right concept is the contravariant derivative for the vector bundle $L$ over a Poisson manifold $(M, \alpha)$ :

$$
D_{w}: \Gamma(L) \rightarrow \Gamma(L), \quad w \in T^{*} M,
$$

such that for any $f \in C^{\infty}(M)$ and $s \in \Gamma(L)$

$$
D_{f w} s=f D_{w} S, \quad D_{w} f s=f D_{w} s+w(\sigma f) s,
$$

where $\sigma=[\alpha,]_{s}$ is the Schouten-Nijenhuis bracket with $\alpha$. 
The sharp map $\sharp: T^{*} M \rightarrow T M$ is defined by $w(\sharp p)=\alpha(p, w)$ with $w, p \in$ $T^{*} M$. It induces a homomorphism on the relevant cohomologies:

$$
\sharp: H_{\mathrm{deR}}(M, \mathbb{R}) \rightarrow H_{\mathrm{LP}}(M, \alpha),
$$

where $H_{\mathrm{deR}}(M, \mathbb{R})$ is de $\operatorname{Rahm}$ cohomology on forms and $H_{\mathrm{LP}}(M, \alpha)$ is Lichnerowicz-Poisson cohomology on contravariant antisymmetric tensors with the differential $\sigma$.

Using a contravariant derivative, the prequantization formula becomes

$$
\hat{f} s=-i \hbar D_{d f} s+f s
$$

and condition (2.1) implies that

$$
C_{D}=-\frac{i}{\hbar} \alpha,
$$

where $C_{D}$ is the curvature of $\mathrm{D}$,

$$
C(w, p) s=D_{w} D_{p} s-D_{p} D_{w} s+D_{[w, p]} s,
$$

with [ , ] the Koszul brackets on one-forms of a Poisson manifold. Thus, the Poisson-Chern class of $L$ is

$$
p c_{1}(L)=-\frac{1}{2 \pi \hbar}[\alpha]
$$

which is the image of the real Chern class $c_{1}(L)$ under (2.7). So, the existence of a prequantization bundle requires that the preimage of $1 /(2 \pi \hbar)[\alpha]$ under $(2.7)$ should be an element of $H^{2}(M, \mathbb{Z})$ (i.e., $H^{2}(M, \mathbb{Z})$ is sent by the inclusion $\mathbb{Z} \subset \mathbb{R}$ to $\left.H_{\mathrm{deR}}^{2}(M, \mathbb{R})\right)$. The converse is also true. Thus, this construction gives a representation of $C^{\infty}(M)$, which is however not always faithful. For further details and relevant concepts, see textbook by Vaisman [27].

To summarize, a Poisson manifold $(M, \alpha)$ has a prequantization bundle iff there exist a vector field $v$ and a closed two-form $w$ that represents an integral cohomology class of $M$ (i.e., $1 /(2 \pi \hbar)[w] \in H^{2}(M, \mathbb{Z})$ ), such that

$$
\alpha=-\sharp w+\mathcal{L}_{v} \alpha .
$$

Within the geometric quantization framework such $(M, \alpha)$ is called quantizable.

We finally show that if the Poisson tensor satisfies (2.12) then it also satisfies (1.7). Let $(X, \eta)$ be a solution of (1.2), for a generic surface $\Sigma$. By direct computation we get the value of the action $(1.1)$ on $(X, \eta)$ as

$$
S_{\mathrm{cl}}(X, \eta)=\int_{\Sigma} X^{*}(w)+\int_{\partial \Sigma} i_{v} \eta
$$

for the generic boundary condition. If we specialize to $\Sigma=\mathbb{S}^{2}$, there is no boundary term and the integrality of $w$ obviously implies (1.7). However, 
the opposite is obviously not true, since condition (1.7) makes sense also when $[\alpha]$ is not in the image of the sharp map.

\section{Gauge transformations of the Poisson sigma model}

In this section, we analyze the role of the integrality condition (1.8) for the Poisson tensor $\alpha$ in the nonperturbative definition of the Kontsevich formula (1.6).

There is a simple argument supporting the need of (1.8). The Kontsevich formula appears from the perturbative expansion around the classical trivial solution $X=x, \eta=0$ of (1.6). One can ask if there exist instantons, e.g., inequivalent nontrivial solutions, around which to expand the integral. The expansion around a classical solution $(X, \eta)$ has the form of an asymptotic series

$$
\mathrm{e}^{(i / \hbar) S_{\mathrm{cl}}(X, \eta)}\left(b_{0}+\hbar b_{1}+\hbar^{2} b_{2}+\cdots\right)
$$

and in general is not just a power series. However, due to boundary conditions (1.5), every solution maps the boundary $\partial D$ to a single point $x$ and defines an algebroid morphism from $T \mathbb{S}^{2}$ to $T^{*} M$. Therefore, property (1.7) implies that the action evaluated on a solution is equal to $2 \pi n \hbar$ and there is no exponential factor in the expansion (3.1). This ensures that the full expansion (i.e., over all nontrivial solutions) of (1.6) will give rise anyway to a formal power series in $\hbar$ (which is correct from the point of view of star products).

In this section, we investigate the role of the integrality condition in the nonperturbative definition of (1.6). In subsection 3.1, we analyze the on-shell gauge transformations of the PSM for a generic closed surface $\Sigma$ and for the disk D with boundary conditions (1.5). In the expansion of the path integral (1.6) around a non-trivial solution, we have to insure that the exponential of the classical action $S_{\mathrm{cl}}$ (i.e., the value of the action functional (1.1) on a classical solution) is well defined. In other words, if two Lie algebroid morphisms $\hat{X}_{1}=\left(X_{1}, \eta_{1}\right)$ and $\hat{X}_{2}=\left(X_{2}, \eta_{2}\right)$ are gauge equivalent, then we must require the following condition:

$$
\mathrm{e}^{(i / \hbar) S_{\mathrm{cl}}\left(X_{1}, \eta_{1}\right)}=\mathrm{e}^{(i / \hbar) S_{\mathrm{cl}}\left(X_{2}, \eta_{2}\right)} .
$$

There are several ways of integrating the infinitesimal on-shell transformations (1.4). The integrality condition (1.8) comes as a necessary condition to satisfy (3.2) in the PSM on the disk with boundary conditions (1.5) if we choose the finite transformations to define a groupoid action of $\mathcal{G}^{D, M}$ see (3.13) and (3.14) for its definition. In subsections 3.2 and 3.3, we perform a formal analysis of the path integral formula (1.6). The picture that 
comes out is consistent with this choice of on-shell gauge transformations; moreover, the integrality condition (1.8) allows us to reduce (1.6) to a quantum mechanical path integral on the symplectic groupoid integrating $M$. For pedagogical reasons, in subsection 3.2, we analyze first the symplectic case where these ideas emerge in a simple way; the analysis is repeated in subsection 3.3 for the general Poisson case, at the cost of a more technical discussion involving the underlying algebroid structures.

Finally observe that we have assumed the Poisson manifold to be integrable. If this is not the case, a topological groupoid $\mathcal{G}(M)$ "integrating" $M$ exists anyway as the reduced phase space of the Poisson sigma model [8]. This groupoid may also be regarded as a stacky Lie groupoid [25] and possibly the considerations of the present section go through. This would imply the integrality condition (1.8) also in the nonintegrable case (notice that the condition is well defined for every Poisson manifold).

\subsection{On-shell gauge transformations}

We start by considering the Poisson sigma model defined over a closed surface $\Sigma$.

A Lie algebroid $A \rightarrow M$ is a vector bundle $A$ over $M$ with a Lie algebra structure (over $\mathbb{R}$ ) on the sections of $A$ and a vector bundle morphism $\rho: A \rightarrow T M$ (called the anchor) which induces a Lie algebra homomorphism from sections of $A$ to vector fields on $M$. The bracket on sections of $A$ is required to satisfy $\left[f e_{1}, e_{2}\right]=f\left[e_{1}, e_{2}\right]-\left(\rho\left(e_{2}\right) f\right) e_{1}$ for all $f \in C^{\infty}(M)$ and $e_{1}, e_{2} \in \Gamma(A)$. A Poisson manifold $(M, \alpha)$ induces a Lie algebroid structure on the cotangent bundle $T^{*} M$ with $\sharp$ as its anchor; as for the Lie bracket on its sections (a.k.a. the Koszul bracket), it is enough to define it on exact oneforms by $[d f, d g] \equiv d\{f, g\}$. The tangent bundle $T \Sigma$ carries a canonical Lie algebroid structure with anchor the identity and the standard Lie bracket. The bundle morphism

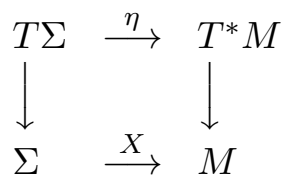

satisfies the equations of motion (1.3) iff $(X, \eta)$ is a Lie algebroid morphism. In other words, the action functional, which is defined for any bundle morphism, is extremized when the morphism is a Lie algebroid morphism. This result follows straightforwardly from the definition of Lie algebroid morphisms given in [13]. Thus, the space of classical solutions of the Poisson sigma model is the space $\operatorname{Mor}\left(T \Sigma, T^{*} M\right)$ of Lie algebroid morphisms. 
A Lie groupoid $(\mathcal{G} \rightrightarrows M, s, t)$ is a smooth manifold $\mathcal{G}$ with surjective submersions $s$ and $t$ from $\mathcal{G}$ to $M$ and a smooth multiplication map from $\mathcal{G}^{(2)}=\{(g, h) \in \mathcal{G} \times \mathcal{G} \mid t(h)=s(g)\}$ to $\mathcal{G}$ making $\mathcal{G}$ into a category in which all elements are invertible. The points in $M$ are simultaneously considered as the objects of the category and the identity morphisms. A Lie groupoid is called source-simply-connected (ssc) if the $s$-fibers are connected and simply connected. The vector bundle $\left.\operatorname{ker}(d s)\right|_{M}$ has a natural structure of a Lie algebroid over $M$ with anchor $d t$ and Lie bracket induced by multiplication. We denote by $A(\mathcal{G})$ the Lie algebroid of the Lie groupoid $\mathcal{G}$. Not all Lie algebroids arise in this way. Those which do are called integrable.

A morphism of Lie groupoids induces a morphism of the corresponding Lie algebroids by taking its differential at the identity sections. Let us recall the second Lie theorem for Lie algebroids:

Theorem 3.1. Let $\mathcal{G}$ and $\tilde{\mathcal{G}}$ be two Lie groupoids integrating the Lie algebroids $A(\mathcal{G})$ and $A(\tilde{\mathcal{G}})$, respectively. If $\mathcal{G}$ is source simply connected (ssc), then for any Lie algebroid morphism $A(\mathcal{G}) \rightarrow A(\tilde{\mathcal{G}})$ there exists a unique integrating Lie groupoid morphism $\mathcal{G} \rightarrow \tilde{\mathcal{G}}$.

For more information on Lie algebroids and groupoids, see $[5,18,19]$.

A Poisson manifold is said to be integrable if the corresponding Lie algebroid $T^{*} M$ is integrable. If this is case, we will denote the corresponding ssc Lie groupoid simply by $\mathcal{G}(M)$. Indeed, $\mathcal{G}(M)$ carries a symplectic structure which is compatible with groupoid multiplication and hence it is called a symplectic groupoid. Let $\Pi(\Sigma) \rightrightarrows \Sigma$ be the fundamental groupoid of $\Sigma$ which is defined as follows: $\Pi(\Sigma)$ consists of homotopy classes $\left[c_{u v}\right]$ of curves $c_{u v}$ in $\Sigma$ starting at $v \in \Sigma$ and ending at $u \in \Sigma$, with $s\left(\left[c_{u v}\right]\right)=v$ and $t\left(\left[c_{u v}\right]\right)=u$, and its multiplication law is induced by concatenation. Observe that for $\Sigma$ simply connected $\Pi(\Sigma)=\Sigma \times \Sigma$. Since $\Pi(\Sigma)$ is the ssc groupoid integrating $T \Sigma$, we can associate to any Lie algebroid morphism (3.3) a unique Lie groupoid morphism

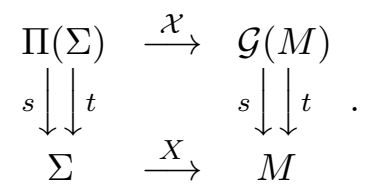

Therefore, we can identify the space $\operatorname{Mor}\left(T \Sigma, T^{*} M\right)$ of Lie algebroid morphisms with the space $\operatorname{Mor}(\Pi(\Sigma), \mathcal{G}(M))$ of Lie groupoid morphisms.

For the case of integrable Poisson manifolds $(M, \alpha)$, we may then describe the space of classical solutions of the Poisson sigma model as $\operatorname{Mor}(\Pi(\Sigma), \mathcal{G}$ 
$(M))$. Next, we would like to introduce the gauge group which naturally acts on this space (i.e., which sends solutions to solutions).

Let us consider the infinite-dimensional groupoid $\mathcal{G}^{\Sigma}=\{\hat{\Phi}: \Sigma \rightarrow \mathcal{G}(M)\}$ over $M^{\Sigma}=\{\Phi: \Sigma \rightarrow M\}$ with structure maps defined pointwise. Namely, we define source and target by $s(\hat{\Phi})(u)=s(\hat{\Phi}(u)), t(\hat{\Phi})(u)=t(\hat{\Phi}(u))$ for $u \in \Sigma$ and multiplication by $\hat{\Phi}_{1} \hat{\Phi}_{2}(u)=\hat{\Phi}_{1}(u) \hat{\Phi}_{2}(u)$. A section $\lambda$ of the associated algebroid $A\left(\mathcal{G}^{\Sigma}\right)$ (this algebroid has been defined, at least for $\Sigma$ one-dimensional, in [4] intrinsically in terms of the Lie algebroid $T^{*} M$, so it exists also for nonintegrable Poisson manifolds) is defined by giving a section $\lambda(\Phi) \in \Gamma\left(\Phi^{*} T^{*} M\right)$ for every $\Phi \in M^{\Sigma}$. There is a natural groupoid action ${ }^{2}$ of $\mathcal{G}^{\Sigma}$ on $\operatorname{Mor}(\Pi(\Sigma), \mathcal{G}(M))$ which is given by

$$
X_{\hat{\Phi}}(u)=t(\hat{\Phi})(u), \quad \mathcal{X}_{\hat{\Phi}}\left(\left[c_{u v}\right]\right)=\hat{\Phi}(u) \mathcal{X}\left(\left[c_{u v}\right]\right) \hat{\Phi}(v)^{-1},
$$

where $(X, \mathcal{X}),\left(X_{\hat{\Phi}}, \mathcal{X}_{\hat{\Phi}}\right) \in \operatorname{Mor}(\Pi(\Sigma), \mathcal{G}(M)), \hat{\Phi} \in \mathcal{G}^{\Sigma}$ with $s(\hat{\Phi})=X$ and $\left[c_{u v}\right]$ is the homotopy class of a curve $c_{u v}$ in $\Sigma$. From (3.5), one can easily deduce the action of $\mathcal{G}^{\Sigma}$ on the space of Lie algebroid morphisms $\operatorname{Mor}\left(T \Sigma, T^{*}\right.$ $M),(X, \eta) \rightarrow\left(X_{\hat{\Phi}}, \eta_{\hat{\Phi}}\right)$. However, the concrete expression is not relevant for our discussion.

One could also consider a group action by introducing the group of bisections of $\mathcal{G}^{\Sigma}$,

$$
\operatorname{Bis}\left(\mathcal{G}^{\Sigma}\right)=\left\{\sigma: M^{\Sigma} \rightarrow \mathcal{G}^{\Sigma} \mid s \circ \sigma=\mathrm{id}, t \circ \sigma=\psi_{\sigma} \in \operatorname{Diff}\left(M^{\Sigma}\right)\right\} .
$$

It is clear that formula (3.5) defines in a straightforward way a group action of Bis $\mathcal{G}^{\Sigma}$ on $\operatorname{Mor}(\Pi(\Sigma), \mathcal{G}(M))$. The orbits of $\mathcal{G}^{\Sigma}$ contain those of Bis $\mathcal{G}^{\Sigma}$ and they coincide only when it is true that for every $\hat{\Phi} \in \mathcal{G}^{\Sigma}$, there exists a bisection $\sigma$ passing through it, e.g., $\sigma(s(\hat{\Phi}))=\hat{\Phi}$. Moreover let us remark that $(\operatorname{Bis} \mathcal{G})^{\Sigma}=\{\hat{\sigma}: \Sigma \rightarrow \operatorname{Bis} \mathcal{G}(M)\}$ is a subgroup of $\operatorname{Bis}\left(\mathcal{G}^{\Sigma}\right)$. Namely, there is a Lie group morphism

$$
\begin{array}{ccc}
\Psi:(\operatorname{Bis} \mathcal{G})^{\Sigma} & \rightarrow & \operatorname{Bis}\left(\mathcal{G}^{\Sigma}\right) \\
\hat{\sigma} & \mapsto & \sigma
\end{array}
$$

with $\sigma(\Phi)(u) \equiv \hat{\sigma}(u)(\Phi(u)), u \in \Sigma, \Phi \in M^{\Sigma}$. We also have a left-inverse map $\widetilde{\Psi}: \sigma \mapsto \hat{\sigma}$ defined by $\hat{\sigma}(u)(m) \equiv \sigma(\underline{m})(u), u \in \Sigma, m \in M$, where $\underline{m}$ denotes the constant map with value $m$. Thus, $\Psi$ is injective.

There is an interesting subset of gauge transformations which can be described as follows. For every map $f: \Sigma \rightarrow \Sigma$, its differential $f_{*}: T \Sigma \rightarrow T \Sigma$

\footnotetext{
${ }^{2}$ This action may actually be extended to the space of maps $\Pi(\Sigma) \rightarrow \mathcal{G}(M)$ that are compatible with $s$ and $t$ maps (i.e., maps for which (3.4) is a commutative diagram), which however are not required to be compatible with multiplication.
} 
is a Lie algebroid morphism. We denote by $\mathcal{F}: \Pi(\Sigma) \rightarrow \Pi(\Sigma)$ the corresponding Lie groupoid morphism. Thus, using a map $f$, any groupoid morphism $(X, \mathcal{X})$ can be transformed to a new groupoid morphism $(X \circ f, \mathcal{X} \circ$ $\mathcal{F})$ as follows:

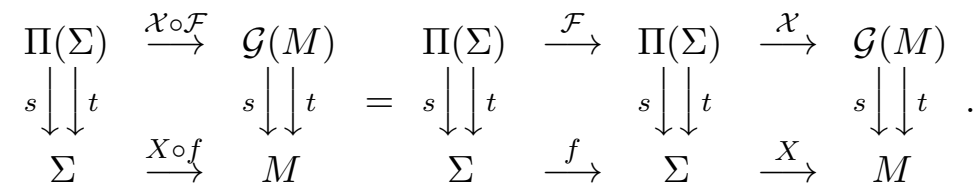

We call a map $f: \Sigma \rightarrow \Sigma$ liftable to $\Pi(\Sigma)$ if there exists a map $F: \Sigma \rightarrow \Pi(\Sigma)$, such that $s \circ F=$ id and $t \circ F=f$. In this case, $\mathcal{F}\left(\left[c_{u v}\right]\right)=F(u)\left[c_{u v}\right] F(v)^{-1}$, so that there exists a gauge transformation $\hat{\Phi}=\mathcal{X} \circ F \in \mathcal{G}^{\Sigma}$ such that

$$
\mathcal{X} \circ \mathcal{F}\left(\left[c_{u v}\right]\right)=\mathcal{X}([F(u)]) \mathcal{X}\left(\left[c_{u v}\right]\right) \mathcal{X}\left(\left[F(v)^{-1}\right]\right)=\mathcal{X}_{\hat{\Phi}}\left(\left[c_{u v}\right]\right) .
$$

Let us comment more on the liftability condition of $f: \Sigma \rightarrow \Sigma$ and give some examples. Consider $\Sigma=\mathbb{S}^{2}$. Then, $\Pi\left(\mathbb{S}^{2}\right)=\mathbb{S}^{2} \times \mathbb{S}^{2}$ and every map $f: \mathbb{S}^{2} \rightarrow \mathbb{S}^{2}$ is liftable with $F(u)=(f(u), u)$. In particular, a constant map $f(u)=u_{0}$ is liftable and thus any groupoid morphism $(X, \mathcal{X})$ is gauge equivalent to a trivial morphism $\left(X\left(u_{0}\right), \mathcal{X}\left(\left[u_{0}, u_{0}\right]\right)\right)$ through (3.7) and (3.8). We will use this property to derive the integrability condition (1.8). Indeed, constant maps are liftable only for $\mathbb{S}^{2}$. Another class of liftable maps, for any $\Sigma$, is the subgroup of diffeomorphisms defined by the group of bisections; namely, for every $F \in \operatorname{Bis}(\Pi(\Sigma))$, we have that $f=t \circ F \in \operatorname{Diff}(\Sigma)$ is liftable by definition. In general, not every diffeomorphism of $\Sigma$ comes from a bisection of $\Pi(\Sigma)$. However, every diffeomorphism connected to the identity is indeed liftable, and so it acts as a finite gauge transformation. This is consistent with having a topological theory. Observe that for $\Sigma$ simply connected, every diffeomorphism is liftable, so every diffeomorphism acts as a finite gauge transformation.

Now we study the value of the action (1.1) on a solution $\hat{X} \in \operatorname{Mor}(T \Sigma$, $T^{*} M$ ). The base map $X$ maps $\Sigma$ to a symplectic leaf $\mathcal{L}$ (see, e.g., the Appendix in [2] for a proof). The tangent space of a leaf $\mathcal{L}$ is spanned by the Hamiltonian vector fields $X_{f}=\{f$,$\} and is endowed with a symplectic$ form defined by

$$
\omega_{\mathcal{L}}\left(X_{f}, X_{g}\right)=\{f, g\}
$$

Thus the action (1.1) evaluated on a solution $\hat{X}$ can be rewritten as the pull-back of the symplectic form $\omega_{\mathcal{L}}$ on the leaf $\mathcal{L} \supset X(\Sigma)$ as

$$
S_{\mathrm{cl}}(X, \eta)=\int_{\Sigma} X^{*}\left(\omega_{\mathcal{L}}\right)
$$

It is important to note that not every map $X: \Sigma \rightarrow \mathcal{L} \subset M$ is the base map of a Lie algebroid morphism, unless $\operatorname{dim} \mathcal{L}=\operatorname{dim} M$. 
Next, we have to require that for two gauge equivalent solutions, condition (3.2) is satisfied. Consider two gauge equivalent solutions, $(X, \eta),\left(X_{\hat{\Phi}}, \eta_{\hat{\Phi}}\right) \in$ $\operatorname{Mor}\left(T \Sigma, T^{*} M\right)$ related by a gauge transformation $\hat{\Phi} \in \mathcal{G}^{\Sigma}$ corresponding to a liftable map $f: \Sigma \rightarrow \Sigma$ as described in (3.7) and (3.8). In its turn, the action functionals on these solutions are related to each other by

$$
S_{\mathrm{cl}}\left(X_{\hat{\Phi}}, \eta_{\hat{\Phi}}\right)=\int_{\Sigma} X_{\hat{\Phi}}^{*}\left(\omega_{\mathcal{L}}\right)=\int_{\Sigma} f^{*} \circ X^{*}\left(\omega_{\mathcal{L}}\right)=\operatorname{deg}(f) S_{\mathrm{cl}}(X, \eta),
$$

where $\operatorname{deg}(f)$ is the degree of the map $f$. For $|\operatorname{deg}(f)|=0,2$, the requirement (3.2) implies the integrality condition

$$
\frac{1}{2 \pi \hbar} S_{\mathrm{cl}}(X, \eta) \in \mathbb{Z}
$$

for any Lie algebroid morphism $(X, \eta)$. Since for $\Sigma=\mathbb{S}^{2}$ every constant map is liftable, we get at condition (1.8).

This result can be easily extended to surfaces with boundary, see the discussion in Section 5. Let us anticipate the case of the disk $D$ with boundary conditions (1.5), which is the relevant case for (1.6). Due to this particular boundary condition, every classical solution is constant on the boundary $\partial D$, $\left.X\right|_{\partial D}=x_{0}$. Therefore, classical solutions on the disk with the given boundary conditions also correspond to Lie algebroid morphism $T \mathbb{S}^{2} \rightarrow T^{*} M$. Thus, the previous discussion for $\mathbb{S}^{2}$ can be applied to this case and we encounter again the same integrality condition (3.12). More precisely, the relevant groupoid for these boundary conditions is

$$
\mathcal{G}^{D, M}=\left\{\hat{\Phi} \in \mathcal{G}^{D} \mid \hat{\Phi}(\partial D) \subset M\right\}
$$

over $M^{D}$; the group of bisections can be described as follows

$$
\operatorname{Bis}\left(\mathcal{G}^{D, M}\right)=\left\{\sigma \in \operatorname{Bis}\left(\mathcal{G}^{D}\right)|\sigma(X)|_{\partial D}=\left.X\right|_{\partial D}\right\} .
$$

It is clear that (3.5) defines an action of $\mathcal{G}^{D, M}$ on the space of groupoid morphisms $(X, \mathcal{X}) \in \operatorname{Mor}(D \times D, \mathcal{G}(M) ; M)$ that are trivial on the boundary: i.e., $\mathcal{X}(\partial D \times \partial D) \subset M$. We refer to Section 5 for a detailed discussion of the case with generic boundary conditions.

In order to conclude that the integrality condition (1.8) is actually a necessary condition, we have to discuss if $\mathcal{G}^{D, M}$ acts off-shell and is really a symmetry of the problem. These are the gauge transformations that look most natural from the geometrical point of view of Lie groupoid theory, but it is not obvious a priori that we are allowed to use them. Since the algebra of infinitesimal transformations closes only on-shell, the first problem one has to face is the correct understanding of the infinitesimal off-shell transformations, see for instance the discussion in [1]. Then, as we already verified on-shell, the integration of the infinitesimal transformations (1.4) 
does not have a unique answer in the same way as the integration of a Lie algebra may correspond to different Lie groups.

Our strategy will be different. We plan to discuss finite off-shell gauge transformations elsewhere, while in the next subsections we will discuss the role of the integrality in the nonperturbative properties of (1.6). We will get a picture that confirms this choice of the gauge transformations for a disk $D$. However we cannot claim anything about the case of a closed surface $\Sigma$, in particular $\mathbb{S}^{2}$.

Finally, we refer to [3] for a discussion of the moduli space of Lie algebroid morphisms divided by finite gauge transformations in the case of a generic surface $\Sigma$.

\subsection{The symplectic case}

In this subsection, we consider the case when the Poisson tensor is invertible. Then $\omega \equiv \alpha^{-1}$ is a symplectic form. In this case, any map $X: \mathbb{S}^{2} \rightarrow$ $M$ defines a Lie algebroid morphism $\hat{X}=(X, \eta): T \mathbb{S}^{2} \rightarrow T^{*} M$ with $\eta_{\mu}=$ $-\omega_{\mu \nu} d X^{\nu}$. Therefore, the integrality condition (1.8) corresponds to the integrality of the pairing between $[\omega / 2 \pi \hbar]$ and $\pi_{2}(M)$ and is weaker than the usual geometric quantization condition $\left[\frac{1}{2 \pi \hbar} \omega\right] \in H^{2}(M, \mathbb{Z})$. These two conditions are the same only if $M$ is simply connected.

Let us interpret this condition in the path integral defined in (1.6). Assume for the moment that the target manifold $M$ is simply connected. In the symplectic case, the formula (1.6) essentially reduces to the original Feynman path integral formula for quantum mechanics. We can formally integrate $\eta$ and arrive to the expression

$$
f * g(x)=\int_{X(\infty)=x} f(X(1)) g(X(0)) \mathrm{e}^{(i / \hbar) \int_{D} X^{*}(\omega)} d X
$$

where one should integrate over any map $X: D \rightarrow M$. While formally integrating $^{3} \eta$ in (1.6) we took into account the boundary conditions (1.5). It is clear that the observable we are integrating depends only on the boundary

\footnotetext{
${ }^{3}$ It is convenient to introduce polar coordinates $(r, \phi)$ on the disk $D(r \leq 1)$. Then $\eta_{\phi}$ is regarded as a Lagrangian multiplier with the boundary condition $\left.\eta_{\phi}\right|_{r=1}=0$. Upon the integration of $\eta_{\phi}$, we get a $\delta$-function in path integral imposing the relation on $\partial_{r} X$ and $\eta_{r}$ on $r<1$.
} 
values $\gamma=\left.X\right|_{\partial D}$ so that, if $\omega$ is exact then (3.15) can be defined as

$$
f * g(x)=\int_{\gamma(\infty)=x} f(\gamma(1)) g(\gamma(0)) \mathrm{e}^{(i / \hbar) \int_{\gamma} d^{-1} \omega} d \gamma,
$$

where the sum is over all loops $\gamma: \mathbb{S}^{1} \rightarrow M$, with $\gamma(\infty)=x$, or, equivalently, as proposed in [7], over trajectories $\gamma: \mathbb{R} \rightarrow M$ with $\gamma( \pm \infty)=x$. Integrality (1.8) allows one to define the weight function also when $\omega$ is not exact. Indeed, we can define the action using the following prescription

$$
S=\int_{\gamma}\left(d^{-1} \omega\right) \equiv \int_{D} X^{*}(\omega)
$$

where $X: D \rightarrow M$ is any map such that $\left.X\right|_{\partial D}=\gamma$. The definition (3.17) is good if it is independent of the choice of $X$. Namely, let us choose two maps $X_{1}$ and $X_{2}$ such that $\left.X_{1}\right|_{\partial \Sigma}=\left.X_{2}\right|_{\partial \Sigma}=\gamma$, then the difference between two definitions is given by

$$
\int_{D} X_{1}^{*}(\omega)-\int_{D} X_{2}^{*}(\omega)=\int_{X_{1}(D)} \omega-\int_{X_{2}(D)} \omega=\int_{c_{2}} \omega
$$

where $c_{2}=X_{1}(D) \cup X_{2}(D)$ is the two-sphere obtained by joining $X_{1}(D)$ and $X_{2}(D)$ along the boundary. In order to define unambiguously the path integral measure, we have to require

$$
\mathrm{e}^{(i / \hbar) \int_{c_{2}} \omega}=1
$$

for any sphere $c_{2}$ in $M$. This is the standard argument which is used for the Dirac charge quantization of $U(1)$ monopole [12].

If $\pi_{1}(M)$ is nontrivial, not every loop $\gamma$ can be covered by a disk $D$, so the path integral in (3.16) is over contractible loops only. If $H_{1}(M)$ is trivial then any loop is the boundary of some surface $\Sigma$, possibly with handles, so that one can extend (3.16) to any loops by covering them with higher genus surfaces. In order to do this, we have to require that $\left[\frac{1}{2 \pi \hbar} \omega\right] \in H^{2}(M, \mathbb{Z})$. However, if $H_{1}(M)$ is nontrivial, the extension of the path integral (3.16) to all loops goes beyond the Poisson sigma model. The associativity of the Kontsevich formula suggests that it is not necessary to include these contributions in order to have an associative $*$-product.

We can make contact with the discussion of the previous subsection by reinterpreting the equivalence suggested by this construction in terms of gauge invariance under finite gauge transformations. Indeed thanks to integrality, the $1 \mathrm{~d}$ path integral (3.16) can be defined as the $2 \mathrm{~d}$ path integral (3.15), and we can consider this construction as a way of getting rid of the gauge equivalence. So, the correct gauge transformation is the one that identifies two maps $X_{i}: D \rightarrow M, i=1,2$, if they coincide on the boundary. 
We consider again $M$ to be simply connected, so that $\mathcal{G}(M)=M \times M$ is the (ssc) groupoid integrating it.

It is immediately clear that the homotopy equivalence is too weak. From the previous discussion, one possible candidate for the gauge group could be $(\operatorname{Bis}(M \times M))^{D}=\left\{\sigma: D \rightarrow \operatorname{Diff}(M)|\sigma|_{\partial D}=\mathrm{id}\right\}$, which integrates the infinitesimal gauge transformations of the model, $\delta X^{\mu}=\alpha^{\mu \nu} \beta_{\nu}$ with $\left.\beta\right|_{\partial D}=$ 0 . In general, also this group will be too small; in fact, take any two sphere in $\mathrm{M}$, divide it in two disks $X_{1}(D)$ and $X_{2}(D)$, we require that it exists $\sigma \in(\operatorname{Bis}(M \times M))^{D}$ such that $X_{2}=\sigma\left(X_{1}\right)$. In particular, let the loop be the point $x$, we require that any class in $\pi_{2}(M, x)$ be represented by $\sigma(x)$. This will be impossible, if for example $\pi_{2}(\operatorname{Diff}(M))=0$, like for $M=\mathbb{S}^{2}$, (see [23]). Finally, let us analyze the groupoid $\mathcal{G}^{D, M}$ defined in (3.14). It clearly acts on $M^{D}$ and it is easy to verify that the source fiber $\mathcal{G}_{X}^{D, M}$ of $X \in M^{D}$ is $\left\{(Y, X)|Y: D \rightarrow M, Y|_{\partial D}=\left.X\right|_{\partial D}\right\}$ so that any two configurations $X_{1}$ and $X_{2}$ coinciding on the boundary will be gauge equivalent.

We should stress that this discussion of the finite gauge transformations is based on the action (3.17). If we accept the formal derivation of (3.15) from (1.6), the instantons of the PSM correspond to those $X \in M^{D}$ such that $\left.X\right|_{\partial D}=x$. Therefore, they are all equivalent and are represented by the constant loop $\gamma=x$ in (3.16). This agrees with the discussion of the previous subsection: the on-shell gauge groupoid $\mathcal{G}^{D, M}$ is extended off-shell only after the formal integration of $\eta_{\phi}$ and there is only one classical solution with the prescribed boundary condition, modulo gauge transformations.

\subsection{The general Poisson case}

The discussion of (1.6) of the previous subsection for the symplectic case can be successfully repeated for a generic integrable Poisson manifold.

Let us start from (1.6) and introduce polar coordinates $(r, \phi)$ on the disk $D$; once that we integrate over the Lagrange multiplier $\eta_{\phi}$, we get

$f * g(x)=\int_{X(\infty)=x} f(X(1)) g(X(0)) \mathrm{e}^{(i / \hbar) \int_{D} d^{2} \sigma \partial_{\phi} X^{\mu} \eta_{r \mu}} \delta\left(\partial_{r} X+\alpha \eta_{r}\right) d X d \eta_{r}$.

Although the Poisson tensor is degenerate, the constraint $\partial_{r} X+\alpha \eta_{r}=0$ can be explicitly solved in the following way. We are going to prove that for any $\left(X, \eta_{r}\right)$ satisfying $X(\infty)=x$ and $\partial_{r} X+\alpha \eta_{r}=0$, there exists a unique algebroid morphism $\left(X, \eta_{r}, \tilde{\eta}_{\phi}\right): T D \rightarrow T^{*} M$ or, equivalently, a map 
$\xi_{X, \eta_{r}}: D \rightarrow \mathcal{G}(M)_{x}$ satisfying $\xi_{X, \eta_{r}}(\infty)=x$, and vice versa. It should be stressed that $\tilde{\eta}_{\phi}$ is determined by $\eta_{r}$ and $X$ and should not be confused with $\eta_{\phi}$. Indeed, for each fixed $\phi \in \mathbb{S}^{1}$, we let $X^{\phi}(r)=X\left(r \mathrm{e}^{i \phi}\right), \epsilon^{\phi}(r)=\eta_{r}\left(r \mathrm{e}^{i \phi}\right)$; it is clear that $\left(X^{\phi}, \epsilon^{\phi}\right)$ is an algebroid morphism from $T I \rightarrow T^{*} M$, and we consider the unique groupoid morphism $\left(X^{\phi}, \mathcal{X}^{\phi}\right): I \times I \rightarrow \mathcal{G}(M)$ that integrates it. Then, we define $\xi: \mathbb{S}^{1} \times I \rightarrow \mathcal{G}(M)_{X(0)}, \xi(\phi, r)=\mathcal{X}^{\phi}(r, 0)$. Since $\xi(\phi, 0)=\mathcal{X}^{\phi}(0,0)=X(0)$, we can consider $\xi: D \rightarrow \mathcal{G}(M)_{X(0)}$, so that it defines a groupoid morphism $D \times D \rightarrow \mathcal{G}(M):(u, v) \rightarrow \xi(u) \xi(v)^{-1}$ and by differentiation, the desired algebroid morphism $(X, \eta): T D \rightarrow T^{*} M$, where $X(u)=t(\xi(u))$ and $\left.\eta\right|_{T_{u} D}=R_{\xi(u)^{-1} *} \circ \xi_{*}$. Finally, $\xi_{X, \eta_{r}}: D \rightarrow \mathcal{G}(M)_{x}$ defined by $\xi_{X, \eta_{r}}(u)=\xi(u) \xi(\infty)^{-1}$ satisfies $\xi_{X, \eta_{r}}(\infty)=x$. The converse statement is at this point obvious. We remark that $\left.\tilde{\eta}_{\phi}\right|_{\partial D}$ is not zero in general, and the on-shell configurations of the PSM correspond to those algebroid morphisms that satisfy boundary conditions (1.5) or, equivalently, to those maps $\xi$ such that $\xi(\partial D)=x$.

The value of the action on these configurations $\left(X, \eta_{r}\right) \leftrightarrow \xi$ can be equivalently written as

$$
\begin{aligned}
S\left(X, \eta_{r}\right) & =\int_{D} \partial_{\phi} X^{\mu} \eta_{r \mu} r d r d \phi=-\int_{D} \alpha^{\mu \nu} \tilde{\eta}_{\phi \mu} \eta_{r \nu} r d r d \phi \\
& =-\int_{D} X^{*}\left(\omega_{\mathcal{L}_{x}}\right)=\int_{D} \xi^{*}(\Omega)=S(\xi),
\end{aligned}
$$

where $\omega_{\mathcal{L}_{x}}$ is the symplectic form of the leaf $\mathcal{L}_{x}$ containing $x$ and $\Omega=t^{*}\left(\omega_{\mathcal{L}_{x}}\right)$ is the symplectic form of the symplectic groupoid restricted to $\mathcal{G}(M)_{x}$.

We can then rewrite (1.6), analogously to (3.15), as a sum over all algebroid morphisms $(X, \eta): T D \rightarrow T^{*} M$, or, equivalently over all $s$-vertical maps $\xi: D \rightarrow \mathcal{G}(M)_{x}$ with $\xi(\infty)=x$,

$$
f * g(x)=\int_{\xi: D \rightarrow \mathcal{G}(M)_{x}, \xi(\infty)=x} f(t(\xi(1))) g(t(\xi(0))) \mathrm{e}^{(i / \hbar) S(\xi)} d \xi .
$$

It is now easy to see that the integrality condition (1.8) plays the same role as in the symplectic case. We recall that (1.8) implies the integrality the symplectic form $\Omega$ over all the spherical cycles contained in $\mathcal{G}(M)_{x}$. If $\alpha$ satisfies (1.8), then $\exp i / \hbar S(\xi)$ depends only on the boundary value $\left.\xi\right|_{\partial D}$. In fact, if $\left.\xi_{1}\right|_{\partial D}=\left.\xi_{2}\right|_{\partial D}$, then $S\left(\xi_{1}\right)-S\left(\xi_{2}\right)=\int_{\xi_{1} \cup \xi_{2}} t^{*}\left(\omega_{\mathcal{L}_{x}}\right) \in 2 \pi \hbar \mathbb{Z}$, where $\xi_{1} \cup \xi_{2}$ is the sphere in $\mathcal{G}(M)_{x}$ obtained by joining $\xi_{1}$ and $\xi_{2}$ along the boundary. By identifying the configurations coinciding on the boundary, we get the final formula

$$
f * g(x)=\int_{\xi: \mathbb{S}^{1} \rightarrow \mathcal{G}(M)_{x}, \xi(\infty)=x} f(t(\xi(1))) g(t(\xi(0))) \mathrm{e}^{(i / \hbar) S(\xi)} d \xi .
$$


Finally, it is clear that, since classical solutions are constant on the boundary, they all correspond to the constant map.

It is useful to remark that one can get formula (3.22) by applying the construction of $\mathcal{G}(M)$ as a Marsden-Weinstein reduction in $T^{*} P M$, as explained in Section 4. In fact, after the formal integration of $\eta_{\phi}$, we can interpret each configuration $\left(X, \eta_{r}\right)$ as a loop $\gamma: \mathbb{S}^{1} \rightarrow \mathcal{C}_{X(0)}$, where $\gamma(\phi)=\left(X^{\phi}, \epsilon^{\phi}\right)$ and $\mathcal{C}_{y}$ denotes the set of algebroid morphisms starting at $y$. Let $\gamma(\phi) \rightarrow \underline{\gamma(\phi)}$ denote the quotient $\mathcal{C} \rightarrow \mathcal{G}(M)$. Then, the above construction means that we identify two loops $\gamma_{1}$ and $\gamma_{2}$ if and only if ${\underline{\gamma_{1}}(\phi)}_{\gamma_{1}(\infty)^{-1}}^{=\gamma_{2}(\phi)}{\underline{\gamma_{2}(\infty)^{-1}}}^{-1}$ for each $\phi \in \mathbb{S}^{1}$.

We can now analyze this construction in terms of the gauge transformations introduced in Section 3. The groupoid $\mathcal{G}^{D, M}$ acts on $\xi: D \rightarrow \mathcal{G}(M)_{x}$ as follows: let $\hat{\Phi} \in \mathcal{G}^{D, M}$ be such that $s(\hat{\Phi})=t \circ \xi$, then we define

$$
\xi_{\hat{\Phi}}(u)=\hat{\Phi}(u) \xi(u), \quad u \in D .
$$

We see that this action coincides with (3.5), where $\mathcal{X}(u, v)=\xi(u) \xi(v)^{-1}$ and $X(u)=t \circ \xi(u)$. Let us verify that this action induces the equivalence relation involved in the path integral construction. In fact, if $\left.\xi\right|_{\partial D}=\left.\nu\right|_{\partial D}$, then we can write $\nu=\xi_{\hat{\Phi}}$ where $\hat{\Phi} \in \mathcal{G}(M)^{D, M}$ is defined by $\hat{\Phi}(u)=\nu(u) \xi(u)^{-1}$.

\section{Prequantization of symplectic groupoids}

In this section, we study the prequantization of the source simply connected (ssc) symplectic groupoid $\mathcal{G}(M)$ of an integrable Poisson manifold $M$. Since the space of units is a Lagrangian submanifold, every prequantization of $\mathcal{G}(M)$ induces a flat structure on $M$. In [29], it has been shown that if $\mathcal{G}(M)$ is prequantizable, then there is a unique prequantization bundle of $\mathcal{G}(M)$ such that this flat structure is trivial. In [11], it has been shown that a necessary and sufficient condition for the symplectic groupoid $\mathcal{G}(M)$ to be prequantizable is that the symplectic form is integer on every $\mathbb{S}^{2} \subset s^{-1}(y)$, for every $y \in M$, where $s$ is the source map. Since any two-sphere in the source fiber defines an algebroid morphism and vice versa, this integrality condition is the same as (1.8).

We develop here an alternative and straightforward approach based on the description of $\mathcal{G}(M)$ as the Marsden-Weinstein symplectic reduction of a cotangent bundle, obtained in [8]. We are going to get the reduction of the prequantization of the cotangent bundle. This will give us an explicit description of the prequantization of $\mathcal{G}(M)$ and, hopefully, will clarify some important issues. 
Let us first recall the construction of the symplectic groupoid $\mathcal{G}(M)$ in [8]. Let us consider the Hamiltonian formulation of the Poisson sigma model over a rectangle $R=[-T, T] \times I$ with coordinates $(t, u)$ labeling time and space, respectively. The boundary conditions are $\eta_{t}=0$ on $[-T, T] \times \partial I$. In this world-sheet geometry, the action (1.1) can be rewritten as

$$
S=\int_{R} d t d u\left[-\left\langle\eta_{u}, \partial_{t} X\right\rangle+\left\langle\eta_{t},\left(\partial_{u} X+\alpha \eta_{u}\right)\right\rangle\right]
$$

The corresponding phase space is given by the space of vector bundle morphisms $T I \rightarrow T^{*} M$ with the symplectic structure defined by the by first term in the action (4.1). This phase space can be interpreted as the cotangent bundle $T^{*} P M$ of the path space $P M$ of maps $I \rightarrow M$ with exact symplectic structure $d \theta$, where $\theta$ is the canonical Liouville form. The field $\eta_{t}$ is a Lagrangian multiplier which enforces the first class constraints

$$
\partial_{u} X+\alpha(X) \eta_{u}=0
$$

that generate a distribution of subspaces spanned by Hamiltonian vector fields. Let $H$ be the group generated by the Hamiltonian vector fields. The constraints (4.2) define a coisotropic infinite dimensional submanifold $\mathcal{C}(M)$ of $T^{*} P M$. The reduced phase space $\mathcal{C}(M)$ of the Poisson sigma model is the set of integral manifolds of this distribution and is obtained as the MarsdenWeinstein symplectic quotient $T^{*} P M / / H=\mathcal{C}(M) / H$. In [8], it has been shown that $\mathcal{C}(M)$ carries the structure of topological groupoid over $M$ and, if smooth, it is the symplectic groupoid $\mathcal{G}(M)$. Alternatively, using the language of Lie algebroids one can give the following description of $\mathcal{G}(M)$ $[10,22]$. Elements of $\mathcal{C}(M)$ are those bundle maps that are also Lie algebroid morphisms $\hat{\gamma}: T I \rightarrow T^{*} M$. Elements of $\mathcal{C}(M)$ are Lie algebroid morphisms modulo homotopy. We say that two algebroid morphisms $\hat{\gamma}_{0}, \hat{\gamma}_{1}: T I \rightarrow T^{*} M$ are homotopic if there exists a Lie algebroid morphism $\hat{\gamma}: T([0,1] \times I) \rightarrow$ $T^{*} M$ such that $\left.\hat{\gamma}\right|_{\{0\} \times I}=\hat{\gamma}_{0},\left.\hat{\gamma}\right|_{\{1\} \times I}=\hat{\gamma}_{1}$ and $\left.\hat{\gamma}\right|_{[0,1] \times \partial I}$ is the zero bundle map (i.e., the boundary conditions (1.5) are satisfied).

Let us recall the relation between symplectic reduction and prequantization in the finite dimensional case following [30]. Consider the cotangent bundle $T^{*} \mathcal{M}$ of a manifold $\mathcal{M}$ with the canonical symplectic structure $\omega=d \theta$; let $\mathcal{C}$ be a coisotropic submanifold of $T^{*} \mathcal{M}$ and $\underline{\mathcal{C}}$ be the reduced phase space with symplectic form $\underline{\omega}$. The cotangent bundle $T^{*} \mathcal{M}$ is prequantized by the trivial line bundle $T^{*} \mathcal{M} \times \mathbb{C}$ with connection $\nabla^{\theta} \equiv d-(i / \hbar) \theta$. Then a sufficient condition for $\underline{\mathcal{C}}$ to be prequantizable is that $\theta$ satisfies the integrality condition

$$
\frac{1}{2 \pi \hbar} \int_{l} \theta \in \mathbb{Z}
$$

for every closed curve $l$ inside a leaf of the characteristic foliation. In fact, we can construct the prequantization line bundle $L(\underline{\mathcal{C}})$ as $\mathcal{C} \times \mathbb{C} / \sim$, where 
$\left(c_{0}, z_{0}\right) \sim\left(c_{1}, z_{1}\right)$ whenever $c_{0}$ and $c_{1}$ are in the same leaf and

$$
z_{1}=z_{0} \mathrm{e}^{i / \hbar \int_{c_{0}}^{c_{1}} \theta}
$$

where $\theta$ is integrated over any path contained in the leaf and connecting $c_{0}$ to $c_{1}$. The integrality (4.3) ensures that the equivalence relation is well defined, i.e., that the exponent in (4.4) does not depend on the concrete choice of a path between $c_{0}$ and $c_{1}$. A section $\psi \in \Gamma(L(\underline{\mathcal{C}}))$ can be identified with an equivariant function $\psi: \mathcal{C} \rightarrow \mathbb{C}$, i.e., a solution to $\nabla_{V}^{\theta} \psi=0$ for every vector field $V$ along the leaves. If $W$ is a vector field on $\mathcal{C}$ that projects to a vector field $W^{\prime}$ on $\underline{\mathcal{C}}$, then $\nabla_{W}^{\theta}$ depends only on $W^{\prime}$ and defines a connection on $\Gamma(L(\underline{\mathcal{C}}))$ whose curvature is $\underline{\omega}$. For further details, we refer to Proposition (8.4.9) in [30]. Although this proposition is proved for the finite dimensional case, its generalization for the infinite dimensional cotangent bundle is straightforward.

In the following proposition, we apply the above construction to our case of $\mathcal{M}=P M, \mathcal{C}=\mathcal{C}(M)$ and $\underline{\mathcal{C}}=\mathcal{G}(M)$.

\section{Proposition 4.1.}

i) The ssc symplectic groupoid $\mathcal{G}(M)$ is prequantizable if and only if $\alpha$ satisfies the integrality condition (1.10) for $c_{2}=\mathbb{S}^{2}$.

ii) The reduction of the prequantization of $T^{*} P M$ induces a trivial flat structure over $M$.

We give an alternative proof to the one in [11].

Proof. Let $\alpha$ satisfy (1.10). The coisotropic leaves of $\mathcal{C}(M)$ are homotopic Lie algebroid morphisms $\hat{\gamma}: T I \rightarrow T^{*} M$, so that a loop contained in a leaf is a Lie algebroid morphism $T\left(S^{1} \times I\right) \rightarrow T^{*} M$. Due to the boundary conditions, the boundaries of the annulus $S^{1} \times I$ are mapped to fixed points, so we effectively deal with a Lie algebroid morphism $\hat{X}=(X, \eta): T \mathbb{S}^{2} \rightarrow$ $T^{*} M$. The integral of the Liouville form $\theta$ is given by the first term of (4.1) and thus on a leaf it coincides with the pairing between $\alpha$ and $\hat{X}$. Therefore the condition (4.3) becomes (1.10), i.e.,

$$
\frac{1}{2 \pi \hbar} \int_{l} \theta=\frac{1}{2 \pi \hbar} S_{\mathrm{cl}}(X, \eta)=\frac{1}{2 \pi \hbar} \ll \alpha,\left(\mathbb{S}^{2}, \hat{X}\right) \gg \in \mathbb{Z},
$$

so that $\mathcal{G}(M)$ is prequantizable.

Conversely, let us suppose that $\mathcal{G}(M)$ is prequantizable with integer symplectic form $\Omega$. Let $\mathcal{C}_{y} \subset \mathcal{C}(M)$ be the subset of those algebroid morphisms starting at $y \in M$ and let $\gamma: \mathbb{S}^{1} \rightarrow \mathcal{C}_{y}$ be defined by $\gamma(t)=(X(t, u), \eta(t, u))$. Then $F(r, t)=(X(t, r u), r \eta(t, r u))$ is a homotopy to the trivial morphism 
$(y, 0)$, i.e., $\mathcal{C}_{y}$ is simply connected for every $y \in M$. Since homotopic morphisms have the same end points, every leaf is contained in $\mathcal{C}_{y}$ for some $y \in M$ and every loop $l$ contained in it can be covered by a disk $D \subset \mathcal{C}_{y}$, such that $\partial D=l$. We then have that $\int_{l} \theta=\int_{D} d \theta=\int_{\mathbb{S}^{2}} \Omega \in 2 \pi \hbar \mathbb{Z}$, so that $\theta$ satisfies (4.3).

Finally, it can be verified that $\left.L(\mathcal{G}(M))\right|_{M}=M \times \mathbb{C}$ and $\nabla^{\theta} \psi=d \psi$, for every $\psi: M \rightarrow \mathbb{C}$; i.e., the flat connection is trivial.

Remark 4.1. We can explicitly describe the line bundle $L(\mathcal{G}(M))$ as $\mathcal{C}(M)$ $\times \mathbb{C} / \sim$, where $\left(\hat{\gamma}_{0}, z_{0}\right) \sim\left(\hat{\gamma}_{1}, z_{1}\right)$ if $\hat{\gamma}_{0}$ and $\hat{\gamma}_{1}$ are homotopic algebroid morphisms and

$$
z_{1}=z_{0} \mathrm{e}^{i / \hbar \ll \alpha,(D, \hat{X}), \gg}
$$

where $\hat{X}: T D \rightarrow T^{*} M$ is the algebroid homotopy between $\hat{\gamma}_{0}$ and $\hat{\gamma}_{1}$ seen as an algebroid morphism from the disk, thanks to the boundary conditions. Because of (4.5), this definition of equivalence does not depend on the concrete choice of $(D, \hat{X})$.

Furthermore we can reinterpret the integrality condition (4.3) using the following path integral argument. Consider a cotangent bundle $T^{*} \mathcal{M}$ with the canonical symplectic form $\omega=d \theta$. We assume that the coisotropic submanifold $\mathcal{C}$ is defined by the first class constraints $\left\{\phi_{i}\right\}$ and let $G$ be the group of diffeomorphisms generated by the constraints. Let $p: \mathcal{C} \rightarrow \underline{\mathcal{C}}=\mathcal{C} / G$ be the canonical projection and let $\underline{\omega}$ be the symplectic form of $\underline{\mathcal{C}}\left(\right.$ i.e., $\left.p^{*} \underline{\omega}=\omega\right)$.

We consider the following action functional

$$
S(\gamma, \lambda)=\int_{\gamma}\left(p \dot{q}-\lambda^{i} \phi_{i}(q, p)\right) d t
$$

The ultimate goal is to define the path integral

$$
\int d \gamma d \lambda \cdots \mathrm{e}^{(i / \hbar) S(\gamma, \lambda)}=\int d \gamma \delta\left(\phi^{i}(q, p)=0\right) \cdots \mathrm{e}^{(i / \hbar) S(\gamma, 0)}
$$

where $\cdots$ stands for some gauge invariant observable. The integration over the $\lambda_{i}$ 's restricts the path integral to $\mathcal{E}=\{\gamma: I \rightarrow \mathcal{C}\}$; the gauge symmetries force us to identify $\gamma(t)$ and $g(t) \gamma(t)$, with $g \in P G=\{g: I \rightarrow G\}$ and so to define the integral over $\mathcal{E} / P G$, which are the maps with values in the reduced phase space $\underline{\mathcal{C}}$. A necessary condition for this to work is that the procedure defines a correct measure over $\mathcal{E} / P G$. 
Let us study in details the measure of the path integral (4.8). Once the integration over $\lambda$ is done, the action can be defined as follows:

$$
S(\gamma) \equiv S(\gamma, 0)=\int_{\gamma} \theta
$$

We need to compare the actions of two gauge equivalent configurations $\gamma$ and $g \gamma, g \in P G$. Namely, we have

$$
\int_{\gamma} \theta-\int_{p_{0}} \theta-\int_{g \gamma} \theta+\int_{p_{1}} \theta=\int_{\Sigma} \omega=\int_{i(\Sigma)} \underline{\omega}=0
$$

where $p_{0}\left(p_{1}\right)$ is a path contained in a leaf and connecting $\gamma(0)(\gamma(1))$ and $g(0) \gamma(0)(g(1) \gamma(1)) ; \Sigma$ is a surface such that $\partial \Sigma=\gamma p_{0}^{-1}(g \gamma)^{-1} p_{1}$. Such $\Sigma$ exists since $G$ is connected so that $g$ is homotopic to identity. From (4.10), we have

$$
\mathrm{e}^{(i / \hbar) S(g \gamma)}=\mathrm{e}^{i / \hbar \int_{p_{1}} \theta} \mathrm{e}^{-i / \hbar \int_{p_{0}} \theta} \mathrm{e}^{(i / \hbar) S(\gamma)},
$$

and if the condition (4.3) is satisfied, then the expression (4.11) does not depend on the concrete choice of paths $p_{0}$ and $p_{1}$, only end points matter. Remark that fixing, the end points of $\gamma$ in the path integral would break gauge invariance. We can now do the integration along the fiber and reduce the integral to $\mathcal{E} / P G$; i.e., we can define the measure

$$
W(\underline{\gamma})=\mathrm{e}^{(i / \hbar) S(\gamma)} \int_{G \times G} d g_{0} d g_{1} \mathrm{e}^{-i / \hbar \int_{\gamma(0)}^{\gamma(0) g_{0}} \theta} \mathrm{e}^{i / \hbar \int_{\gamma(1)}^{\gamma(1) g_{1}} \theta} .
$$

Vice versa, in order to separate integrations along and transverse to a fiber in (4.3), one needs the integrality condition.

The present argument based on path integral is not a rigorous one. However, in our view, it offers a nice physical intuition behind the integrality condition (4.3). Although we stated this for a quantum mechanical system (i.e., with finite dimensional $T^{*} \mathcal{M}$ ), it is straightforward to generalize it for a Poisson sigma model where the cotangent bundle and the coisotropic submanifold are infinite dimensional.

\section{$5 \quad D$-branes and integrality}

In the previous sections, we discussed the Poisson sigma model over $\Sigma$ either without boundary or with the specific boundary conditions given in (1.5). However, there exist more general non-symmetry-breaking boundary conditions, as discussed in [9], which correspond to coisotropic submanifolds of $M$. A submanifold $\mathcal{D}$ of $M$ is called coisotropic if $\sharp N^{*} \mathcal{D} \subset T \mathcal{D}$, where $N^{*} \mathcal{D}$ 
is the conormal bundle of $\mathcal{D}$. The boundary conditions corresponding to the coisotropic submanifold $\mathcal{D}$ are

$$
X(\partial \Sigma) \subset \mathcal{D},\left.\quad \eta_{t}\right|_{\partial \Sigma} \in \Gamma\left(X^{*}\left(N^{*} \mathcal{D}\right)\right),\left.\quad \beta\right|_{\partial \Sigma} \in \Gamma\left(X^{*}\left(N^{*} \mathcal{D}\right)\right),
$$

and (1.5) corresponds to the case when $\mathcal{D}=M$. Observe that $\sharp N^{*} \mathcal{D}$ defines an involutive distribution on $\mathcal{D}$ (the characteristic foliation) and the gauge transformations correspond to diffeomorphisms along the leaves.

The results of both Sections 3 and 4 can be generalized for the case of a generic coisotropic brane. In following two subsections, we sketch the construction.

\subsection{On-shell gauge transformations with branes}

In analogy with the discussion in subsection 3.1 we define an on-shell gauge group and deduce an integrality condition for the classical action.

Consider a surface $\Sigma$ with $n$ boundary components, $\partial \Sigma=\bigcup_{i=1}^{n} \mathbb{S}_{i}^{1}$ and associate to $\partial \Sigma$ a set $\mathcal{C}=\left\{C_{i}\right\}$ of $n$ coisotropic submanifolds of $M$. The conormal subbundles $N^{*} C_{i}$ are Lie subalgebroids of $T^{*} M$ with anchor map $\sharp: N^{*} C_{i} \rightarrow T C_{i}$. Assuming the Poisson manifold $M$ to be integrable, there are Lagrangian Lie subgroupoids $\mathcal{G}\left(C_{i}\right) \subset \mathcal{G}(M)$ that integrate the Lie subalgebroids $N^{*} C_{i}$ [6]. As we said, the boundary conditions are defined by a choice of $\mathcal{C}$, i.e., $X: \mathbb{S}_{i}^{1} \rightarrow C_{i}$ and $\left.\eta\right|_{\mathbb{S}_{i}} \in \Gamma\left(T^{*} \mathbb{S}_{i}^{1} \otimes X^{*}\left(N^{*} C_{i}\right)\right)$. A classical solution of the equations of motion with these boundary conditions is given by a Lie algebroid morphism $\hat{X}=(X, \eta): T \Sigma \rightarrow T^{*} M$ such that its restriction on the $i$-th component of the boundary $\left.(X, \eta)\right|_{\mathbb{S}_{i}^{1}}$ is a Lie algebroid morphism from $T \mathbb{S}_{i}^{1}$ to $N^{*} C_{i}$. We denote the space of such Lie algebroid morphisms by $\operatorname{Mor}\left(T \Sigma, T^{*} M ; N^{*} \mathcal{C}\right)$. As a consequence of the integrability of the Poisson manifold $M$, we have that this space is the same as the space $\operatorname{Mor}(\Pi(\Sigma), \mathcal{G}(M) ; \mathcal{G}(\mathcal{C}))$ of groupoid morphisms $(X, \mathcal{X})$ such that $\mathcal{X}\left(\left.\Pi(\Sigma)\right|_{\mathbb{S}_{i}^{1}}\right) \subset \mathcal{G}\left(C_{i}\right)$, where $\left.\Pi(\Sigma)\right|_{\mathbb{S}_{i}^{1}}=\left\{\left[c_{u v}\right] \in \Pi(\Sigma) \mid c_{u v} \subset \mathbb{S}_{i}^{1}\right\}$ is the subgroupoid of $\Pi(\Sigma)$ integrating the $i$-th boundary component $\mathbb{S}_{i}^{1}$.

In analogy with the closed case, we define the groupoid $\mathcal{G}^{\Sigma, \mathcal{C}}=\{\hat{\Phi}: \Sigma \rightarrow$ $\left.\mathcal{G}(M) \mid \hat{\Phi}\left(\mathbb{S}_{i}^{1}\right) \subset \mathcal{G}\left(C_{i}\right) \forall i\right\}$ over $M^{\Sigma, \mathcal{C}}=\left\{\Phi: \Sigma \rightarrow M \mid \Phi\left(\mathbb{S}_{i}^{1}\right) \subset C_{i} \forall i\right\}$. Take $(X, \mathcal{X}) \in \operatorname{Mor}(\Pi(\Sigma), \mathcal{G}(M) ; \mathcal{G}(\mathcal{C}))$ and $\hat{\Phi} \in \mathcal{G}^{\Sigma, \mathcal{C}}$ with $s(\hat{\Phi})=X$, then the groupoid action of $\mathcal{G}^{\Sigma, \mathcal{C}}$ on $\operatorname{Mor}(\Pi(\Sigma), \mathcal{G}(M) ; \mathcal{G}(\mathcal{C}))$ is defined by

$$
\mathcal{X}_{\hat{\Phi}}\left(\left[c_{u v}\right]\right)=\hat{\Phi}(u) \mathcal{X}\left(\left[c_{u v}\right]\right) \hat{\Phi}(v)^{-1}
$$


As in the closed case, we can describe part of the gauge groupoid through liftable maps. A map $f: \Sigma \rightarrow \Sigma$ preserving the boundary (i.e., $f\left(\mathbb{S}_{i}^{1}\right) \subset$ $\left.\mathbb{S}_{i}^{1}\right)$ is said to be liftable to $\Pi(\Sigma)$ if there exists a map $F: \Sigma \rightarrow \Pi(\Sigma)$ such that $\left.F\left(\mathbb{S}_{i}^{1}\right) \subset \Pi(\Sigma)\right|_{\mathbb{S}_{i}^{1}}, s \circ F=$ id and $t \circ F=f$. Then $\mathcal{F}\left(\left[c_{u v}\right]\right)=F(u)\left[c_{u v}\right]$ $F(v)^{-1}$ is the corresponding groupoid morphism and it is easy to verify that $(X \circ f, \mathcal{X} \circ \mathcal{F}) \in \operatorname{Mor}(\Pi(\Sigma), \mathcal{G}(M) ; \mathcal{G}(\mathcal{C}))$. Moreover, there exists $\hat{\Phi} \in \mathcal{G}^{\Sigma, \mathcal{C}}$ such that $\mathcal{X} \circ \mathcal{F}=\mathcal{X}_{\hat{\Phi}}$ with $\hat{\Phi}=\mathcal{X} \circ \mathcal{F}$.

Let us concentrate on the case of the disk $D$ with boundary condition defined by the coisotropic submanifold $C$. The corresponding ssc groupoid is $\Pi(D)=D \times D$ and any map $f: D \rightarrow D$ preserving the boundary is liftable to $D \times D$. Considering a constant map $f(u)=u_{0} \in \partial D$, we conclude that any groupoid morphism $(X, \mathcal{X})$ is gauge equivalent to the trivial one $(X(u)=$ $\left.X\left(u_{0}\right), \mathcal{X}\left(\left[c_{u v}\right]\right)=\mathcal{X}\left(\left[u_{0}, u_{0}\right]\right)\right)$. As before, we can use the argument (3.11) to deduce the necessary integrality condition

$$
\frac{1}{2 \pi \hbar} S_{\mathrm{cl}}(X, \eta) \in \mathbb{Z}
$$

for every $(X, \eta) \in \operatorname{Mor}\left(T D, T^{*} M ; N^{*} \mathcal{C}\right)$. Indeed this integrality condition has cohomological interpretation since it depends only on a class $[(\alpha, 0)] \in$ $H_{P L}^{\bullet}(M, \mathcal{D}, \alpha)$ in the appropriate relative cohomology, see the Appendix, for the construction. Using the pairing between relative (co)homology groups introduced in the Appendix, we can write the integrality condition as

$$
\frac{1}{2 \pi \hbar} \ll(\alpha, 0),\left(D, \hat{X}, \partial D,\left.\hat{X}\right|_{\partial D}\right) \gg \in \mathbb{Z},
$$

where $\hat{X}: T D \rightarrow T^{*} M$ and $\left.\hat{X}\right|_{\partial D}: \partial D \rightarrow N^{*} \mathcal{D}$ are Lie algebroid morphisms. If $(\alpha, 0)$ is the trivial element in $H_{\mathrm{PL}}^{\bullet}(M, \mathcal{D}, \alpha)$, then the classical action is zero.

Condition (5.4) is a generalization of condition (4.5) (or condition (1.10) for $\Sigma=\mathbb{S}^{2}$ ). In the case $\mathcal{D}=M$, the condition (5.4) is equivalent to (4.5).

Finally, if $M$ is symplectic with exact symplectic form $\omega=d \theta$, then condition (5.4) is equivalent to Woodhouse's condition (4.3).

\subsection{Reduced phase space picture}

One can consider the reduced phase picture for the case of generic coisotropic branes. We can apply the logic from Section 4 to the case of general boundary conditions. Namely, consider the Poisson sigma model over rectangle $R=[-T, T] \times I$ and impose on the boundary $\partial I=\{0,1\}$ the boundary conditions (5.1) corresponding to the coisotropic submanifolds $\mathcal{D}_{0}$ and 
$\mathcal{D}_{1}$, respectively. Following [9], we consider the reduced phase space with these boundary conditions. We denote by $\mathcal{C}\left(M ; \mathcal{D}_{0}, \mathcal{D}_{1}\right)$ the submanifold of $\mathcal{C}(M)$ where the base maps are paths connecting $\mathcal{D}_{0}$ to $\mathcal{D}_{1}$. It turns out that $\mathcal{C}\left(M ; \mathcal{D}_{0}, \mathcal{D}_{1}\right)$ is coisotropic in $T^{*} P M$. The characteristic foliation on $\mathcal{C}\left(M ; \mathcal{D}_{0}, \mathcal{D}_{1}\right)$ may move the end points of the base maps only along the characteristic foliations of $\mathcal{D}_{0}$ and $\mathcal{D}_{1}$. The leaf space $\mathcal{C}\left(M ; \mathcal{D}_{0}, \mathcal{D}_{1}\right)$ corresponds to a reduced phase space of the model on the annulus with the prescribed boundary conditions. If smooth, $\mathcal{C}\left(M ; \mathcal{D}_{0}, \mathcal{D}_{1}\right)$ is endowed with a symplectic structure. Using the logic from Section 4 , we can study the condition (4.3) in the present setup and construct the prequantization line bundle of $\underline{\mathcal{C}\left(M ; \mathcal{D}_{0}, \mathcal{D}_{1}\right)}$.

In particular, consider the case $\mathcal{D}_{1}=M$. Then the space $\mathcal{C}\left(M ; \mathcal{D}_{0}, M\right)$ (if smooth) is prequantizable if the integrality condition (5.4) is satisfied. The proof is completely analogous to the one presented in Section 4.

\section{Concluding remarks}

The integrality condition (1.8) appears to be a generalization of different integrality conditions within the geometric quantization program. In particular, it points toward the program of quantizing Poisson manifolds through their corresponding symplectic groupoids. In fact, condition (1.8) is a necessary and sufficient condition for the symplectic groupoid to be prequantizable. On the other hand, using the Poisson sigma model as a tool, in this paper we showed that (1.8) plays different roles in the program of giving a nonperturbative meaning to the Kontsevich formula.

These two facts are strictly related and we believe that they will contribute in giving a unified description of the quantization program. In fact, within quantum field theory, it is a textbook fact that the path integral of a gauge invariant theory should be properly defined over its reduced phase space. While for a generic field theory it is very hard to find an explicit description of it, for the Poisson sigma model there is the nice geometric interpretation of reduced phase space as the symplectic groupoid. We believe that the role of the integrality condition (1.8) is to reduce the problem of the nonperturbative definition of (1.6) to that of defining a quantum mechanical path integral for the symplectic groupoid and at the ultimate end in quantizing it.

Many interesting problems arise. First of all, it will be interesting to analyze the role of integrality in the BV action introduced in [7], and hopefully to confirm the picture provided in this paper on these more solid grounds. 
Second, it will be interesting to understand if the reduced phase picture can also help in understanding more complicated observables and in particular on closed surfaces.

\section{Acknowledgements}

We are grateful to Domenico Seminara, Marco Zambon, Chenchang hu, Thomas Strobl and Alexei Kotov for useful discussions. M.Z. thanks INFN Sezione di Firenze and Zurich University where part of this work was carried out. F.B. thanks Zurich University. The research of M.Z. was supported by EU-grant MEIF-CT-2004-500267. A.C. acknowledges partial support of SNF Grant no. 200020-107444/1 and the IHES for hospitality.

\section{A Appendix}

Let $c_{k}=\left[a_{0}, \ldots, a_{k}\right]$ be a $k$-simplex in $\mathbb{R}^{n}$ and let $G$ be an abelian group (for us $G$ will be either $\mathbb{R}$ or $\mathbb{Z}$ ). Consider the space

$$
C_{k}(M ; G)=\left\{\sum_{g_{\alpha} \in G} g_{\alpha}\left(c_{k \alpha}, \hat{X}_{\alpha}\right)\right\}
$$

of finite formal combinations of Lie algebroid morphisms $\hat{X}_{\alpha}: T c_{k \alpha} \rightarrow T^{*} M$ with values in $G$ with boundary operator

$$
\partial\left(c_{k}, \hat{X}\right)=\left(\partial c_{k},\left.\hat{X}\right|_{\partial c_{k}}\right)
$$

Observe that $\left.\hat{X}\right|_{\partial c_{k}}$ is automatically a Lie algebroid morphism $T\left(\partial c_{k}\right) \rightarrow$ $T^{*} M$ and that the extension of $\partial$ to all $C_{k}$ by linearity obviously satisfies $\partial^{2}=0$. Thus, we associate a homology $H_{\bullet}(M ; G)$ to the Poisson manifold $M$. The definition of the cohomology $H^{\bullet}(M ; G)$ with the adjoint coboundary operator is straightforward. Let us show that $H^{\bullet}(M, \mathbb{R})=H_{\mathrm{LP}}^{\bullet}(M, \alpha)$, the usual Lichnerowicz-Poisson cohomology.

Any Lie algebroid morphism $\hat{X}=(X, \eta): T c_{k} \rightarrow T^{*} M$ induces the map

$$
\hat{X}^{*}: \Gamma\left(\wedge^{\bullet} T M\right) \rightarrow \Gamma\left(\wedge^{\bullet} T^{*} c_{k}\right)
$$

and thus we can define the following pairing between $A \in \Gamma\left(\wedge^{k} T M\right)$ and the chain $\left(c_{k}, \hat{X}\right)$

$$
\ll A,\left(c_{k}, \hat{X}\right) \gg \equiv \int_{c_{k}} \hat{X}^{*}(A)=\int_{c_{k}} A^{\mu_{1} \cdots \mu_{k}} \eta_{\mu_{1}} \wedge \cdots \wedge \eta_{\mu_{k}},
$$


where $A$ can be seen as a real valued $k$-cochain; the following lemma proves that the Poisson differential $[\alpha, \cdot]_{s}$ is the adjoint coboundary to $\partial$.

Lemma A.1. Let $A \in \Gamma\left(\wedge^{k-1} T M\right)$ and $\hat{X}=(X, \eta): T c_{k} \rightarrow T^{*} M$ be a Lie algebroid morphism. Then we have

$$
\ll[\alpha, A],\left(c_{k}, \hat{X}\right) \gg=\ll A, \partial\left(c_{k}, \hat{X}\right) \gg .
$$

Proof. Starting from the coordinate expression of the Schouten bracket, it is possible to compute that

$$
[\alpha, A]^{\mu_{1} \cdots \mu_{k}} \eta_{\mu_{1}} \wedge \cdots \wedge \eta_{\mu_{k}}=-d\left(A^{\mu_{1} \cdots \mu_{k-1}} \eta_{\mu_{1}} \wedge \cdots \wedge \eta_{\mu_{k-1}}\right),
$$

where one should use the equations (1.3), i.e., the fact that $\hat{X}=(X, \eta)$ is a Lie algebroid morphism $T c_{k} \rightarrow T^{*} M$.

Indeed this construction works for any Lie algebroid $E$ over $M$ and (A.5) is an analog of Stockes' theorem for a Lie algebroid, where $[\alpha, \cdot]_{s}$ is replaced by the Lie algebroid differential $\delta_{E}$ and $A \in \Gamma\left(\wedge^{k-1} E^{*}\right)$. In fact, every morphism of Lie algebroids $\Phi: E_{1} \rightarrow E_{2}$ induces a chain map $\Phi^{*}:\left(\Gamma\left(\Lambda^{\bullet} E_{2}^{*}\right), \delta_{E_{2}}\right) \rightarrow\left(\Gamma\left(\Lambda^{\bullet} E_{1}^{*}\right), \delta_{E_{1}}\right)$ and the corresponding map $\left[\Phi^{*}\right]$ in cohomology. So, in particular, a morphism $\Phi: T c \rightarrow E$ induces a map $\left[\Phi^{*}\right]: H_{\delta_{E}}^{\bullet} \rightarrow H_{\text {de Rham }}^{\bullet}(c)$.

If we fix a submanifold $i: \mathcal{D} \hookrightarrow M$, then there is a notion of relative (co)homology. A $k$-chain in $(\mathcal{D}, M)$ is a linear combination of pairs $\left(c_{k}, \sigma_{k-1}\right)$, where $c_{k}$ is a $k$-chain in $M$ and $\sigma_{k-1}$ is a $(k-1)$-chain in $\mathcal{D}$. The boundary operator is defined by $\partial\left(c_{k}, \sigma_{k-1}\right)=\left(\partial c_{k}+(-1)^{k} \sigma_{k-1}, \partial \sigma_{k-1}\right)$. Consider also pairs $\left(q_{k}, w_{k-1}\right)$ of forms, $q_{k} \in \Omega^{k}(M)$ and $w_{k-1} \in \Omega^{k-1}(\mathcal{D})$ with coboundary operator $d\left(q_{k}, w_{k-1}\right)=\left(d q_{k}, d w_{k-1}-(-1)^{k} i^{*} q_{k}\right)$. There exists a natural pairing

$$
\left\langle\left(q_{k}, w_{k-1}\right),\left(c_{k}, \sigma_{k-1}\right)\right\rangle=\int_{c_{k}} q_{k}+\int_{\sigma_{k-1}} w_{k-1}
$$

with the property

$$
\left\langle d\left(q_{k}, w_{k-1}\right),\left(c_{k+1}, \sigma_{k}\right)\right\rangle=\left\langle\left(q_{k}, w_{k-1}\right), \partial\left(c_{k}, \sigma_{k-1}\right)\right\rangle .
$$

This construction gives rise to relative (co)homology groups $H_{\bullet}(M, \mathcal{D}, \mathbb{R})$ and $H^{\bullet}(M, \mathcal{D}, \mathbb{R})$. There are also integer versions of these groups.

Indeed, the above construction can also be generalized to the case of a Lie algebroid with a fixed subalgebroid. Let us sketch the construction. We are interested in the Lie algebroid $T^{*} M$ of a Poisson manifold $(M, \alpha)$ 
and to subalgebroids corresponding to coisotropic submanifolds. For each coisotropic submanifold $\mathcal{D}$ of $M$, we consider the Lie algebroid morphism

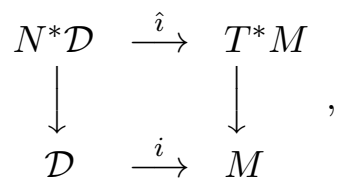

where $\hat{\imath}$ and $i$ are injective immersions. We consider linear combinations of quadruples $\left(c_{k}, \hat{X}, \sigma_{k-1}, \hat{Y}\right)$, where $\hat{X}: T c_{k} \rightarrow T^{*} M$ and $\hat{Y}: T \sigma_{k-1} \rightarrow N^{*} \mathcal{D}$ are Lie algebroid morphisms. We define a boundary operator by

$$
\partial\left(c_{k}, \hat{X}, \sigma_{k-1}, \hat{Y}\right)=\left(\partial c_{k},\left.\hat{X}\right|_{\partial c_{k}}, \partial \sigma_{k-1},\left.\hat{Y}\right|_{\partial \sigma_{k-1}}\right)+(-1)^{k}\left(\sigma_{k-1}, \hat{\imath} \circ \hat{Y}, 0,0\right)
$$

and arrive to a relative homology $H_{\bullet}(M, \mathcal{D})$ associated to $\left(N^{*} \mathcal{D}, T^{*} M\right)$. A $\partial$-closed chain $\left(c_{k}, \hat{X}, \sigma_{k-1}, \hat{Y}\right)$ corresponds to the case when $\partial c_{k}=(-1)^{k-1}$ $\sigma_{k-1}$ and $\left.\hat{X}\right|_{\partial c_{k}}=\hat{Y}$. We also consider linear combinations of pairs $\left(A_{k}\right.$, $\left.v_{k-1}\right), A_{k} \in \Gamma\left(\wedge^{k} T M\right)$ and $v_{k-1} \in \Gamma\left(\wedge^{k-1} N \mathcal{D}\right)$ and define a coboundary operator by

$$
\delta\left(A_{k}, v_{k-1}\right)=\left(\left[\alpha, A_{k}\right], \delta_{N^{*} \mathcal{D}} v_{k-1}-(-1)^{k} \hat{\imath}^{*} A_{k}\right) .
$$

This defines the relative Lichnerowicz-Poisson cohomology $H_{\mathrm{LP}}^{\bullet}(M, \mathcal{D}, \alpha)$. The natural pairing

$$
\ll\left(A_{k}, v_{k-1}\right),\left(c_{k}, \hat{X}, \sigma_{k-1}, \hat{Y}\right) \gg=\left\langle\left(\hat{X}^{*}\left(A_{k}\right), \hat{Y}^{*}\left(v_{k-1}\right)\right),\left(c_{k}, \sigma_{k-1}\right)\right\rangle,
$$

defined through the pairing (A.7), has the property

$$
\ll \delta\left(A_{k}, v_{k-1}\right),\left(c_{k+1}, \hat{X}, \sigma_{k}, \hat{Y}\right) \gg=\ll\left(A_{k}, v_{k-1}\right), \partial\left(c_{k+1}, \hat{X}, \sigma_{k}, \hat{Y}\right) \gg,
$$

which shows that $\delta$ is adjoint to $\partial$.

\section{References}

[1] M. Bojowald, A. Kotov and T. Strobl, Lie algebroid morphisms, Poisson sigma models, and off-shell closed gauge symmetries, arXiv:math.dg/0406445.

[2] F. Bonechi and M. Zabzine, Poisson sigma model over group manifolds, J. Geom. Phys. 54 (2005), 173; arXiv:hep-th/0311213.

[3] F. Bonechi and M. Zabzine, Lie algebroids, Lie groupoids and TFT, to appear in J. Geom. Phys., arXiv:math.sg/0512245.

[4] P. Bressler and A. Chervov, Courant algebroids, arXiv:hep-th/ 0212195 . 
[5] A. Cannas da Silva and A. Weinstein, Geometric models for noncommutative algebras, Berkeley Mathematics Lecture Notes, 10. Amer. Math. Soc., Providence, RI; Berkeley Center for Pure and Applied Mathematics, Berkeley, CA, 1999. xiv + 184 pp.

[6] A. S. Cattaneo, On the integration of Poisson manifolds, Lie algebroids, and coisotropic submanifolds, Lett. Math. Phys. 67 (2004), 33-48.

[7] A. S. Cattaneo and G. Felder, A path integral approach to the Kontsevich quantization formula, Commun. Math. Phys. 212 (2000), 591-611; arXiv:math.qa/9902090.

[8] A. S. Cattaneo and G. Felder, Poisson sigma models and symplectic groupoids, quantization of singular quotients, (eds. N. P. Landsman, M. Pflaum and M. Schlichenmeier), Progr. Math. 198 (2001), 41-73; arXiv:math.SG/0003023.

[9] A. S. Cattaneo and G. Felder, Coisotropic submanifolds in Poisson geometry and branes in the Poisson sigma model, Lett. Math. Phys. 69 (2004), 157-175; arXiv:math.qa/0309180.

[10] M. Crainic and R. L. Fernandes, Integrability of Lie brackets, Ann. Math. 157 (2003), 575-620; arXiv:math.DG/0105033.

[11] M. Crainic and C. Zhu, Integrability of Jacobi structures, arXiv:math.DG/0403268.

[12] P. A. M. Dirac, Quantised singularities in the electromagnetic field, Proc. Roy. Soc. Lond. A 133 (1931), 60.

[13] P. J. Higgins and K. Mackenzie, Algebraic constructions in the category of Lie algebroids, J. Algebra. 129(1) (1990), 194-230.

[14] N. Ikeda, Two-dimensional gravity and nonlinear gauge theory, Annals Phys. 235 (1994), 435; arXiv:hep-th/9312059.

[15] A. A. Kirillov, Elements of the theory of representations, Grundlehren der Mathematischen Wissenschaften, Band, 220, Springer-Verlag, Berlin, 1976, xi + 315 pp.

[16] M. Kontsevich, Deformation quantization of Poisson manifolds, I, Lett. Math. Phys. 66 (2003), 157; arXiv:q-alg/9709040.

[17] B. Kostant, Quantization and unitary representations. I. Prequantization. Lectures in modern analysis and applications, III, pp. 87-208. Lecture Notes in Math., 170, Springer, Berlin, 1970.

[18] K. Mackenzie, Lie groupoids and Lie algebroids in differential geometry, Lond. Math. Soc. Lect. Note Ser., 124, Cambridge University Press, Cambridge, 1987. xvi +327 pp.

[19] I. Moerdijk and J. Mrčun, Introduction to foliations and Lie groupoids, Cambridge Stud. Adv. Math., 91, Cambridge University Press, Cambridge, 2003. $\mathrm{x}+173$ pp. 
[20] P. Schaller and T. Strobl, Poisson structure induced (topological) field theories, Mod. Phys. Lett. A 9 (1994), 3129; arXiv:hep-th/9405110.

[21] P. Schaller and T. Strobl, Introduction to Poisson sigma-models, arXiv:hep-th/9507020.

[22] P. Severa, Some title containing the words 'homotopy' and 'symplectic', e.g. this one, arXiv:math.SG/0105080.

[23] S. Smale, Diffeomorphisms of the 2-sphere, Proc. Amer. Math. Soc., 10 (1959), 621.

[24] J.-M. Souriau, Structure des systèmes dynamiques, Maitrises de mathématiques Dunod, Paris, 1970, xxxii + 414 pp.

[25] H.-H. Tseng and C. Zhu, Integrating Poisson manifolds via stacks, arXiv:math.DG/0411370.

[26] I. Vaisman, On the geometric quantization of Poisson manifolds, J. Math. Phys. 32(12) (1991), 3339-3345.

[27] I. Vaisman, Lectures on the geometry of Poisson manifolds, Progr. Math., 118. Birkhäuser Verlag, Basel, 1994. viii + 205 pp.

[28] A. Weinstein, Noncommutative geometry and geometric quantization, in Symplectic geometry and mathematical physics (Aix-en-Provence, 1990), Progr. Math. 99, Birkhäuser, Boston, MA, 1991, 446-461.

[29] A. Weinstein and P. Xu, Extensions of symplectic groupoids and quantization, J. Reine Angew. Math. 417 (1991), 159-189.

[30] N. Woodhouse, Geometric quantization, Second edition. Oxford Mathematical Monographs, Oxford Science Publications, The Clarendon Press, Oxford University Press, New York, 1992, xii + 307 pp. 